\title{
A Review of Plasmonic Photonic Crystal Fiber
}

\author{
Zahraa Ibrahim AL-Kinani ${ }^{1}$ and Firas Faeq K. Hussain ${ }^{1}$ \\ ${ }^{1}$ Physics Department, College of Science, Al-Muthanna University. \\ "Corresponding author: firas.f@mu.edu.iq
}

Received 25/8/2020 , Accepted 11/10/2020 , published:14/10/2020

DOI: $10.52113 / 2 / 07.02 .2020 / 38-66$

\begin{abstract}
Nanophotonics technology is the study of the confinement of electromagnetic fields on a subwavelength scale and surface plasmons plays a major role in advancing this arising field. They can take various forms, ranging from freely propagating electron density waves along metal surfaces to localized electron oscillations on metal nanoparticles. The interaction between the free electrons' oscillations and electromagnetic waves of light gives the surface plasmons their appealing characteristics. Their ability of light confinement and propagation through subwavelength structures grants the construction of photonic devices with minimum size, hence the characterize and design of plasmonic devices can be resolved using numerical simulation. This review starts with general introduction about Plasmonic photonic crystal fiber. This is followed by a historical background and literature review of Plasmonic PCF. Some types of designs are illustrated. Finally, the applications of Plasmonic PCF are mentioned dependent on the types of design including the circular plasmonic photonic crystal fiber technique.
\end{abstract}

(C) 2020 Al-Muthanna University. All rights reserved

Key words: plasmons, Nanophotonics technology, Plasmonic photonic crystal fiber, PCF, subwavelength structures.

\section{Introduction}

Photonic crystal fibers (PCFs) generally involve two-dimensional array of hollow channels running along the entire length of a glass strand. In a PCF with a solid glass core the guidance by total internal reflection is possible when the average refractive index is lower in the cladding [1]. By presenting twofold symmetry in the area close to the core, the solid-core PCFs may also render biref ringent [2]. The birefringence of such PCFs could be as ten folds higher and 100 times less temperature-sensitive than conventional polarization-maintaining (PM) fibers [3]. The optical 
characteristics of PCFs can be altered by filling the hollow channels with materials such as semiconductors and metals $[3,4]$. Using a high-temperature pressure-cell method, a high purity metal nanowire arrays with length-to-diameter ratios of 100000 can be formed in PCFs [5]. When the core-mode phase matches a surface plasmon (SP) mode on the wires, dips in transmission can be observed. Using a multipole method, theoretical studies showed that the low core-mode attenuation is achievable by suitable design [6,7]. PCFs have advantages over conventional fibers, such as infinite single-mode operation, ultrahigh nonlinearity, and controllable dispersion [8]. Moreover, the polarization characteristics of the PCFs can be altered by filling the cladding holes and hollow core with polymer [9], oil [10], or liquid crystal [11,12]. Light couplers [13,14], filters [15], and amplifiers [16] can be created by fabricating and manipulating the structure of some PCF devices with specific structures. In the past few years, metal nanowire-filled PCFs fabricated by fiber drawing [17] and high-temperature pressure-cell [18] methods have been demonstrated with substantial polarization-dependent transmissions that can be used as sensors, polarizers, and in-fiber devices [19-23].

Because of their ability to confine strongly the light at the sub wavelength scale, the field of plasmonic in photonic crystal fiber has unleashed new research ideas and device concepts [24]. This is cannot be achieved using regular dielectric waveguides due to the diffraction limit [25]. Plasmonic devices like sensors, power dividers, and frequency splitters are of the prime interested applications [26-28]. Metals such as gold and silver have been used to fill either a single air hole or multiple air holes at several locations. Interesting plasmonic behavior known as plasmonic PCF arise, we can turn the PCF to operate as a coupler [22-33].

One of the vital photonic devices is the PCF coupler. It is considered as the basic constituent for upcoming optical networks. The understanding of multicore, particularly, dual-core PCF, opens up new ideas for designing the PCF couplers [34-37], wavelength multiplexers and de multiplexers [38], polarization splitters [39], narrow band pass filters [40] and sensors [41]. For example, dual-core PCF (DC-PCF) 
couplers have several advantages over the conventional optical couplers i.e. easier to design with shorter coupling lengths. Furthermore, the optical characteristics including dispersion profile can be simply controlled as required. In this subject, sun et al. have created a surface plasmon polarization splitter based on DC-PCF with metallic wire. The coupling features of a dual-core hybrid plasmonic photonic crystal fiber is presented and analyzed by using full-victorial finitedifferent method [42].

Plasmonics and the recent birth of metamaterials [43,44] are driving the development of many novel devices with extraordinary functionalities. Due to an applied electromagnetic field, generally the plasmon phenomenon in optical and telecommunication frequencies initiates from the collective oscillations of free charges in a material, plasmonic devices typically require metallic components, which have plenty of free electrons. The negative real permittivity results from these free electrons, which is which essential property of any plasmonic material. However, metals are overwhelmed by large losses, particularly in the visible and ultra-violet (UV) spectral ranges, arising in part from interband electronic transitions. Even the metals with the highest conductivities undergoes major losses at optical frequencies $[45,46]$. These losses negatively affect the performance of plasmonic devices, seriously restricting the viability of several plasmonic applications. However, even the best gain materials available are hardly enough to compensate the losses in the metal. Alternative plasmonic materials with lower losses are essential to develop robust plasmonic devices, because the losses are inherent to the component materials. Lower losses in such enhanced plasmonic components can be readily compensated with available gain materials. Plasmonics could have a magnificent influence on applications at telecommunication and optical frequencies, therefore we will begin this review with an investigation of several possible plasmonic materials [47].

Surface plasmon resonance (SPR) technology as an optical technique has been successfully and broadly used in optical sensing [48,49], optical imaging [50,51], and polarization beam splitter $[52,53]$. When the core guided mode and the surface plasmon polariton (SPP) mode interfaces the face matching 
condition in the metal-filled PCF, the energy of core guided mode can be coupled into the metal layer surface. Spittle et al. successfully presented the micron-sized metal nanowires into silica fiber by blocking particular holes of PCF and filling them with liquid gold $[54,55]$.

\section{History of Plasmonic photonic crystal fiber}

Kao and Hockham (1966) [45], [56] anticipated practical data transmission using optical fibers with reduced attenuation for the first time. This achievement marked an era were optical fibers could bema realistic communication medium. The first experimental realization of low loss optical fibers where in 1970. The photonic bandgap effect, which is an extension to the idea of band structure in semiconductors (a foundation on which modern electronics relies on) to photonics have been predicted for the first time by John and Yablonovitch in (1987). A crystalline lattice creates a potential with periodic modulation which in turn will interact with the electrons creating the electronic band structure. In 1991, Yablonovitch et al. reported the observation of the first bandgap effect (in the micro transition ranges) experimentally in a high index bulk material with hole of $1 \mathrm{~mm}$ in diameter [57]. The manufacture of a novel type of optical waveguide has been investigated by J. C. Knight et al in 1996 . The pure silica core of a photonic crystal fiber is surrounded by air photonic crystalline silica with a hexagonal symmetry [58]. T. A. Birks et al. manufactures an optical fiber made wholly of silica in 1997, by implanting a central core in a twodimensional photonic crystal with a micrometer there are a six columns with air holes [59]. In 2000, J. C. Knight et al. informed the measure of group velocity dispersion for multiple attributes airsilica photonic crystal fibers with abnormal group-velocity dispersion at visible and near-infrared wavelengths. Using the results of this report, they calculated the parameters of the fiber that are needed to form intended dispersion characteristic [60]. In 2001, Kazunori Suzuki et al. high birefringence polarization and low losses photonic crystal fiber has been invented. By taking advantage of numerical simulation were well explained the polarization properties (birefringence, PMD, GVD) [61]. 
The examination of a controlled lateral mode of vertical-cavity surfaceemitting lasers in photonic crystals of two dimensions has been performed by Nouriyuki Yokouchi et al. in 2003. They conclude an imbedded device with defects of single-point and seven point with a relatively large operation in limited mode [62]. In 2003, P. I. Borel et al. investigate the features of polarized light in the structure of a planar photonic crystal waveguide. It shows a photonic bandgap for the TE polarized light and high coupling for the TM polarized light in a simple photonic crystal coupler. Based on this research the formation of structures with three-dimensional finite difference light were successfully modeled [63]. The fabrication of a new optical switch created from photonic crystal has been performed by Takuro Yasuda et al in 2005. The switch consists of a triangular lattice of liquid crystal filled holes. They introduce a novel type switch of photonic crystal coupler depending on the decoupling nature [64]. A. Hassani, M. Skorobogatiy in 2006, investigated the concept of a Microstructured Optical Fiber-based surface Plasmon Sensor with enhanced microfluidics. In this sensor, plasmons on the inner surface of large metallized channels containing analyte could be excited by a fundamental model of a single mode microstructure fiber [65]. Fabio Di and Christopher D. Brooks, developed a gain-staged master power amplifier generator source including an Yb-doped with 100 micrometer -core rode- like photonic crystal fiber based on the final amplifier in 2007. The perfect candidate to occupy the "missing link" between mass and fiber based sold state technologies is the rod like PCF since it has a very large core [66]. In 2008, experimental results on the coupling to surface plasmonic modes on gold nanowires selectively entered into polarization-protecting photonic crystal fibers performed by H. W. Lee et al. For some wavelength scale imaging, the fibers that are filled with gold can be used as a near field tips, beside it can also be used as filter and polarizers [7], [22]. Hung-Ta Chien et al. examined the coupling between dielectric waveguide and a photonic crystal waveguide in 2009. The characteristics of each coupler have been studied both experimentally and theoretically [67]. Shailendra K. Varshney et al. exposed a novel multicore design of photonic crystal fiber in 2009 
via two approaches, finite element and finite element propagation. The analysis of the coupled mode gives power coupling features very compatible with the power estimated by the propagation method [33]. The polarization characteristics has been tested by choosing a photonic crystal fiber filled with metal wires into the cladding core through a full vector model and estimate the result using the finite-element method by Akira Nagasaki et al. in 2011. It is found out that the shift in the resonant wavelength of the created metal-filled cores is shorter than the simulated results and it is due to the higher thermal expansion coefficient [21]. P. Li and J. Zaho in 2013, explained the polarizationdependent coupling by selectively a dualcore photonic crystal fiber filled with gold metal wires in the air holes. Based on this research certain devices can be designed such as polarizers, beam splitter and polarization filter [23]. The numerical investigation of single cores embedded with multiple gold nanowires in triple cores classified in collinear and non collinear has been performed by Susobhan Das et al. in 2013. Magnificent features were attainable via the numerical simulation such as band pass, band rejection filter characteristics along with division capabilities of the designed plasmon PCF coupler device [32]. In 2014, Jitendra Narayan Desh and Rajan Jha introduced a PCF sensor based on graphene and surface plasmon resonance. Graphene aids in the elimination of oxidation of the plasmonic active metal which is a layer of silver. The birefringence nature of the structure permits on component of the core guiding mode to be more sensitive. High amplitude sensitivity and high resolution were displayed by the proposed sensor [49]. Based on the hybrid-surface plasmon resonance, Lei Chen et al. in 2014 anticipated a novel singlepolarization photonic crystal fiber splitter. Numerical simulation of this study shows that the proposed splitter gives a single polarization in the twocommunication window for only several hundred microns in length [68]. In 2015, Zhenkai Fan et al. investigate two kinds of new plasmonic sensor with high sensitivity of refractive index based on an analyst-filled photonic crystal fiber AFPCF. The design with silver is more appropriate and their sensitivities are almost identical because the designed FOM of the AF-PCF with the gold layer 
is much less than that with the silver layer [55]. Using the finite element approach, Abdul Khaleque et al. in 2015 studied the main properties of a dual-core plasmonic photonic crystal fiber. The finite element method can be used to split the incoming signal into two orthogonal polarization states. Based on the outcome of this research, the main properties of the splitter such as bandwidth, dispersion, extinction ratio and splice device [69]. B. M. Younis et al investigate the major characteristics of a dual-core hybrid plasmonic liquid crystal for the PCF in 2016. The possibility to fabricate MVXDEMUX PCF has been achieved with coupling lengths of 753 micrometer shorter than conventional optical fiber [42]. Wee Lit Ng et al.in 2017 examined a highly sensitive novel diamond ring fiber (DRF)-based surface plasmon resonance (SPR) sensor for refractive index sensing. The finite element method has-been used to study the light guiding features and sensing performances numerically. Maximum wavelength can be obtained from the introduced sensor and any amplitude interrogation sensitivity of $6000 \mathrm{~nm} / \mathrm{RIU}$ and $508 \mathrm{RIU}$, respectively, over the refractive index of 1.33-1.39 [70]. In 2018, Sujan Chakma et al. demonstrate numerically a surface plasmon resonance (SPR) biosensor according to a dual-polarized spiral photonic crystal fiber (PCF). The active plasmonic material used is a chemically stable gold which is placed on the outer player of the PCF to facilitate particle manipulation. Using the finite element method, numerical investigation showed that the proposed biosensor has a maximum wavelength sensitivity of 4600 $\mathrm{nm} / \mathrm{RIU}$ and $4300 \mathrm{~nm} / \mathrm{RIU}$ in $\mathrm{x}$ - and $\mathrm{y}$ polarized modes at an analyze refractive index of 1.37. enhanced sensing resolution and suitable linearity features due to high sensitivity. The proposed dual-polarized spiral PCF can be implemented for the detection of biological analytes, organic chemicals, biomolecules, and other unknown analytes [71]. Mohd Fahmi Azman et al. in 2019, proposed a broadband polarization filter based on copper-filled photonic crystal fiber (CFPCF). The anticipated fiber is fabricated by the conventional stack-and-draw method. The properties of the (CFPCF) are studied numerically by considering the image of the cross-sectional scanning electron microscopy. The researchers noticed that the magnitude of cross talk reached up to 
-206 dB over $0.8 \mathrm{~mm}$ length with a broadband width of $282 \mathrm{tnm}$ at a central wavelength of $1790 \mathrm{~nm}$. Moreover, the polarization features of the CFPCF such as cross talk, central wavelength, and band width can be modified by changing the diameter of the copper wire [72].

The numerical investigation of single cores embedded with multiple gold nanowires in triple cores classified in collinear and non collinear has been performed by Susobhan Das et al. in 2013. Magnificent features where attainable via the numerical simulation such as band pass, band rejection filter characteristics along with division capabilities of the designed plasmon PCF coupler device [32]. In 2014, Jitendra Narayan Desh and Rajan Jha introduce a PCF sensor based on graphene and surface plasmon resonance. Graphene aids in the elimination of oxidation of the plasmonic active metal which is a layer of silver. The birefringence nature of the structure permits on component of the core guiding mode to be more sensitive. High amplitude sensitivity and high resolution were displayed by the proposed sensor [49]. Based on the hybrid-surface plasmon resonance, Lei Chen et al. in 2014 anticipated a novel single-polarization photonic crystal fiber splitter. Numerical simulation of this study show that the proposed splitter gives a single polarization in the twocommunication window for only several hundred microns in length [68]. In 2015, Zhenkai Fan et al. investigate two kinds of new plasmonic sensor with high sensitivity of refractive index based on an analyst-filled photonic crystal fiber AFPCF. The design with silver is more appropriate and their sensitivities are almost identical because the designed FOM of the AF-PCF with the gold layer is much less than that with the silver layer [55]. Using the finite element approach, Abdul Khaleque et al. in 2015 studied the main properties of a dual-core plasmonic photonic crystal fiber. The finite element method can be used to split the incoming signal into two orthogonal polarization states. Based on the outcome of this research, the main properties of the splitter such as band width, dispersion, extinction ratio and splice device [69]. B. M. Younis et al investigate the major characteristics of a dual-core hybrid plasmonic liquid crystal for the PCF in 2016. The possibility to fabricate MVXDEMUX PCF has been achieved with coupling lengths of 753 micrometer 
shorter than conventional optical fiber [42]. Wee Lit Ng et al.in 2017 examined a highly sensitive novel diamond ring fiber (DRF)-based surface plasmon resonance (SPR) sensor for refractive index sensing. The finite element method has been used to study the light guiding features and sensing performances numerically. Maximum wavelength can be obtained from the introduced sensor and an amplitude interrogation sensitivity of $6000 \mathrm{~nm} / \mathrm{RIU}$ and $508 \mathrm{RIU}$, respectively, over the refractive index of 1.33-1.39 [70]. In 2018, Sujan Chakma et al. demonstrate numerically a surface plasmon resonance (SPR) biosensor according to a dual-polarized spiral photonic crystal fiber (PCF). The active plasmonic material used is a chemically stable gold which is placed on the outer player of the PCF to facilitate particle manipulation. Using the finite element method, numerical investigation showed that the proposed biosensor has a maximum wavelength sensitivity of 4600 $\mathrm{nm} / \mathrm{RIU}$ and $4300 \mathrm{~nm} / \mathrm{RIU}$ in $\mathrm{x}$ - and $\mathrm{y}$ polarized modes at an analyte refractive index of 1.37. enhanced sensing resolution and suitable linearity features due to high sensitivity. The proposed dual-polarized spiral PCF can be implemented for the detection of biological analytes, organic chemicals, biomolecules, and other unknown analytes [71]. Mohd Fahmi Azman et al. in 2019, proposed a broadband polarization filter based on copper-filled photonic crystal fiber (CFPCF). The anticipated fiber is fabricated by the conventional stack-and-draw method. The properties of the (CFPCF) are studied numerically by considering the image of the cross-sectional scanning electron microscopy. The researchers noticed that the magnitude of cross talk reached up to -206 dB over $0.8 \mathrm{~mm}$ length with a broadband width of $282 \mathrm{tnm}$ at a central wavelength of $1790 \mathrm{~nm}$. Moreover, the polarization features of the CFPCF such as cross talk, central wavelength, and band width can be modified by changing the diameter of the copper wire [22], [72].

\section{Types of Designs}

\subsection{Design of a dual-core plasmonic (PCF) polarization splitter $[1,8,13,30]$}

The DC-PCF cross section consists of a hexagonal lattice with 5 layers of air holes. It can split the incident light into two orthogonal polarization states as shown in Fig. 1a. The lattice constant of the structure is $\Lambda$ and cores $\mathrm{A}$ and $\mathrm{B}$ are 
constructed by erasing two air holes. four holes are increased around cores A and B, (as shown in Fig. 1a, holes with diameters d1), though four other holes (with diameters d2) are decreased [22].

The two innermost circular air holes in the structure are altered to an elliptical air holes by manipulating the diameters of the air holes along the y axis with an ellipticity defined by e $=L_{x} / L_{y}$, where $L_{x}$ and $L_{y}$ are the major and minor axes diameters of the elliptical holes. Finally, two air holes along the perpendicular direction are selectively packed with gold wires (see Fig. 1a for details) with a diameter $d_{m}(=\mathrm{d})$ [5], [6].

The background material is pure silica, and its material dispersion is given by Sellmeier's formula [73]. The relative permittivity of gold is given by the modified Drude-Lorentz model for metal [74] as,

$\varepsilon_{m=} \varepsilon_{\infty}-\frac{\omega_{D}^{2}}{\omega(\omega-j \gamma D)}-\frac{\Delta \varepsilon . \Omega_{L}}{\left(\omega^{2}-\omega_{L}^{2}\right)-j \Gamma_{L \omega}}$

where $\varepsilon_{m}$ is the relative permittivity of the metal, $\varepsilon_{\infty}$ is the relative high frequency permittivity, $\Delta \varepsilon$ is a weighting factor, $\omega$ is the angular frequency of the incident light, $\omega_{D}$ and $\gamma_{D}$ are the plasma and damping frequencies, $\Omega_{L}$ and $\Gamma_{\mathrm{L}}$ represent the frequency and the spectral width of the Lorentz oscillator [18], [22]. A superimposition of even and odd modes can designate the modes in the DC-PCF as can be noted in Fig. 1b. In Fig. 1b, the arrows reveal the direction of the polarization, distinctly showing the parity of even modes and the directions of the $\mathrm{x}$-polarized and $\mathrm{y}$-polarized modes. The coupling lengths of different polarization states are defined as [75],

$$
L_{x, y}=\frac{\lambda}{2\left(n_{x, y-n_{x, y}^{\text {even }}}^{\text {even }}\right)}
$$

The coupling length ratio (CLR) is defined as [76],

$$
\mathrm{CLR}=\frac{L_{y}}{L_{x}}
$$

It is presumed that the input power $P_{\text {in }}$ is pumped into core $\mathrm{A}$, the output power $\left(P_{\text {out }}\right)$ of core $\mathrm{A}$ in $\mathrm{x}$ - and $\mathrm{y}$ - polarized states can be determined as [75],

$$
P_{\text {out }}^{x, y}=P_{\text {in }} \cos ^{2}\left(\frac{\pi L}{2 L_{x, y}}\right)
$$

Likewise, the output power $\left(P_{\text {out }}\right)$ of core $\mathrm{B}$ in $\mathrm{x}-$ and $\mathrm{y}$ polarized states can be calculated as [75], 
$P_{\text {out }}^{x, y}=P_{\text {in }} \sin ^{2}\left(\frac{\pi L}{2 L_{x, y}}\right)$

Since all power for $\mathrm{x}$-polarization remains in core $\mathrm{A}$, it is a vital idea to choose $L$, whereas most of the $y$ polarization power is transferred to core B or vice versa, it is appropriate to have CLR as either $2\left(L_{y}>L_{x}\right)$ or $1 / 2\left(L_{y}<L_{x}\right)$. Besides that, for the two polarization states to produce ultra-broadband splitter, it is beneficial to have relatively flat coupling lengths which will not be very sensitive to the wavelength. In this article, due to $L_{y}>L_{x}, C L R=2$ is used to design the polarization splitter [45], [69].

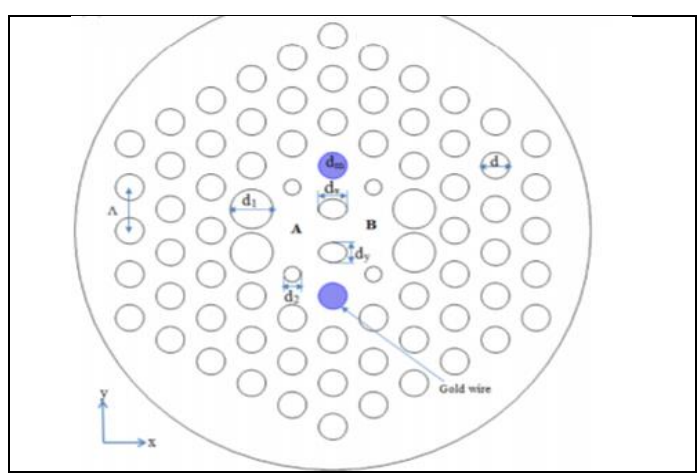

(a)

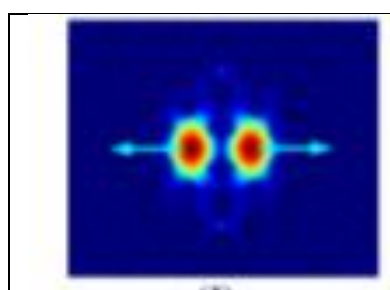

(I)

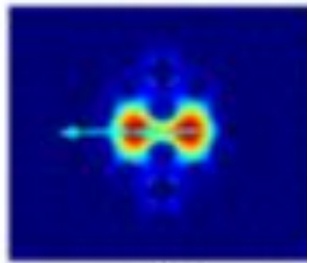

(II) (b)

Fig. (1): (a) Cross section of the proposed gold-filled DC-PCF and (b) description of the electric field profile for the modes in the structure: (I) odd mode of $x$-polarization (II) even mode of $x$-polarization (III) odd mode of $y$-polarization (IV) even mode of $y$ polarization [69].

\subsection{Design of multicore (PCF)based on} $1 \times 4$ power splitter

Fig. 2 illustrates the proposed multicore PCF design. The air holes with diameter $d$ and pitch constant $\Lambda$ construct the cladding where single missing air holes from several locations create multicores. In the shown designs, the numbers from 1 to 5 denote the five cores that has been created. A single optical power is launched at core 1 , which is the central core. Cores 2 and 4 are positioned horizontally, while the cores 3 and 5 are aligned vertically. The locations of the cores are selected in such a manner that the symmetry of the structure does not break up, which makes the problem simpler, i.e., we can use one quarter section of the proposed multicore PCF structure to estimate the coupling characteristics. It should be mentioned that the distance between the cores 1 and 2 and the cores 1 and 3 is different. Hence, the coupling coefficients for cores 1-2 and cores 1-3 are different. For the power transfer to be equivalent in the neighboring cores, the horizontal and 
vertical coupling coefficients must be equal [27], [30].

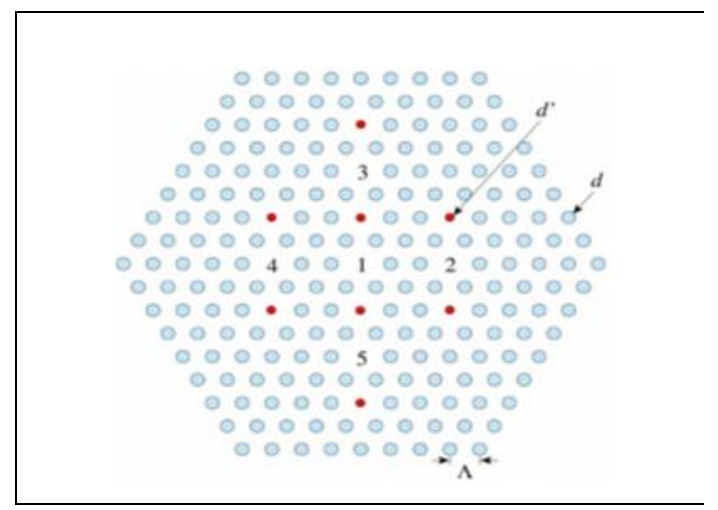

Fig.(2). Schematic view of multicore PCFbased $1 \times 4$ power [33].

This can be accomplished by increasing the vertical coupling coefficient. To predict the equal coupling coefficients for horizontal and vertical coupling, we decrease the diameter of air holes (denoted by $d^{\prime}$ ) located just above and below each core, as shown by red circles in Fig. 3. The size of small air hole can be attained and enhanced when the horizontal and vertical coupling coefficients become equivalent. The refractive index of background material silica is 1.45 in all numerical calculations, the material dispersion of the silica has been neglected in the numerical calculations because the proposed fiber splitter has small length (few $\mu \mathrm{m})$ and focused to operate over C-band. However, for short wavelength operation (e.g., at $800 \mathrm{~nm}$ ), the wavelength dependency of the silica should be considered. Fig. 3 shows the modal field distribution of the $x$-component of the electric field $\left(E_{x}\right)$ at early stage, where the power is pumped into the central core and at the last stage, where the whole power is equally divided into the outer cores. Fig. 3(a) displays the modal field distribution at $1550 \mathrm{~nm}$ wavelength at. After launching the modal field into the central core, the coupling starts and transfer of the power initiated with an equal splitting into neighboring outer cores, as shown in Fig. 3(b) [33], [45].

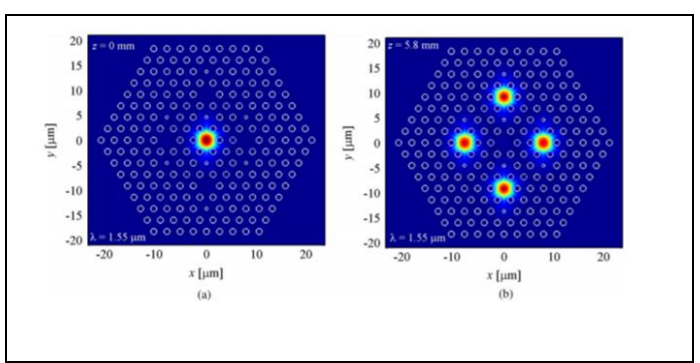

Fig. (3): Modal field distribution( $x$ compenent)of the multicore $\operatorname{PCF}(\mathrm{D} / \Lambda=0.45, \mathrm{~d} / \Lambda=0.23$, and $\Lambda=2.64 \mathrm{~mm}$ )coupler (a) at $\mathrm{z}=0 \mathrm{~mm}$, and $(\mathrm{b})$ at $\mathrm{z}=5.8 \mathrm{~mm}$ at $1550 \mathrm{~nm}$ wavelength [33].

\subsection{Design of splitter based on hybrid- surface plasmon resonance}

The cross-section of the proposed PCF SPWS is shown in Fig. 4. The radius of the background air hole is denoted by "r0"-"r4", the upper hole coated by Au, the air channel in the upper Au layer, the lower hole coated $\mathrm{Au}$, and the air channel 
in the lower Au layer, respectively. Four ellipses are arranged in the Centre of the PCF lattice, which is helpful for producing the birefringence and the letters "a" and "b" are the semi-minor axis and semi-major axis of the ellipse. $\wedge_{\mathrm{ax}}, \wedge_{\mathrm{by}}, \wedge_{0 \mathrm{x}}$, and ${ }^{\wedge}{ }_{0 \mathrm{y}}$ are the hole pitches of the ellipse air hold and background air hole in the $\mathrm{x}$ - and $\mathrm{y}$-axes, respectively. The different materials are represented by different colors, as shown in the legend of Fig. 4. Finally, a perfectly matched layer (PML) is used to match the outmost layer [33], [34]. [36]

The background material is made of pure silica and its dispersion is determined by the Sell-meier equation, where the optical constants are based on experimental results [74]. For precise calculation, the material dispersion of $\mathrm{Au}$ is determined by the Drude-Lorentz model and its optical constants are based on empirical results. The modal loss (defined in decibels per meter) can be defined as:

$\left.\propto=40 \pi \operatorname{Im}\left(n_{e f f}\right) /(\ln (10) \lambda)\right)$

where $\lambda$ is the optical wavelength, and $\operatorname{Im}\left(n_{\text {eff }}\right)$ is the imaginary part of the effective modal refractive index.

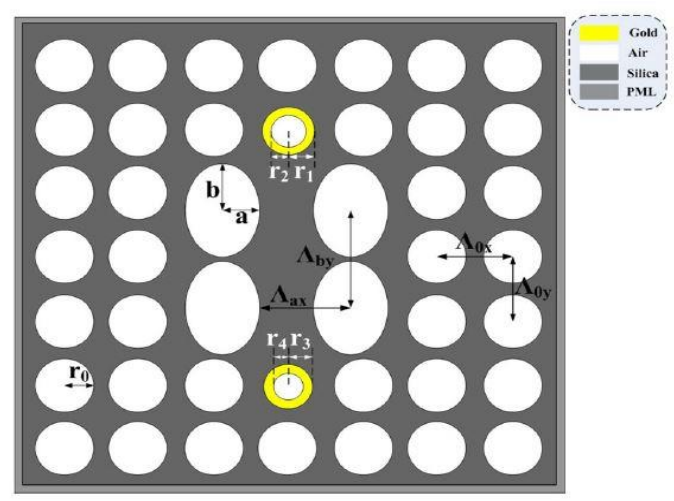

Fig. (4): Cross-section of the proposed PCF SPWS [68].

The hybridized modes are frequently called odd- and even mode, but the presented SWPS has two wavelengths required to be processed, thus for suitability we name the four modes $\mathrm{S} 1$, S2, S3, and S4 respectively. Based on Fig. 5., S1, S2, S3, and S4 have the same $\mathrm{x}$ - and $\mathrm{y}$-axis electric field in the core section. The electromagnetic (EM) wave, based on the transmission line theory, will choose the medium with the lowest transmission loss [68].

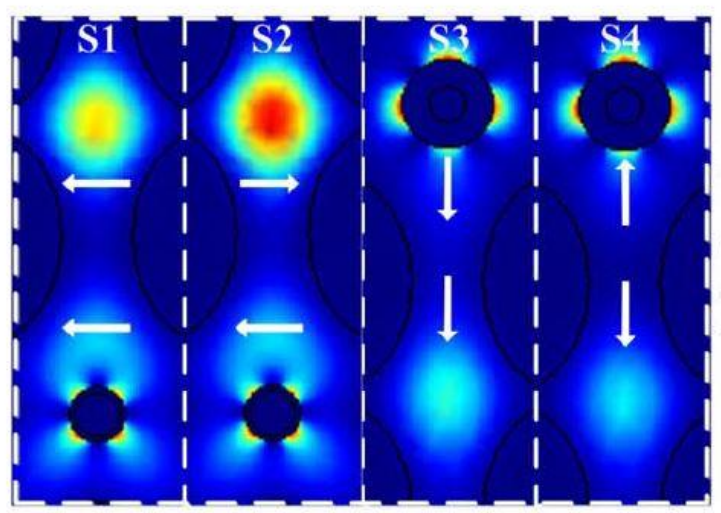

Fig. (5): Four hybridized modes (modal field) [68]. 


\subsection{Design of SPR Sensors}

The cross section of the considered SPR sensors with gold and silver layers are shown in Fig. 6(a). which can be obtained by the perfectly developed stack-and-draw fabrication process. Three layer of air holes arranged in a triangular lattice constitute the designed AF-PCF. The lattice constant $\Lambda$ is $2 \mu \mathrm{m}$. The center small air channel is penetrated by a liquid analyte, which produces the fiber-core of the designed AF-PCF. Two large air holes around the fiber-core are gilded by a- $40 \mathrm{~nm}$ - thickness of gold or silver layer whose remaining blue air channel is also infiltrated into the liquid analyte. In addition, the diameter of blue channel of $\mathrm{d}_{0}$ is $0.5 \mu \mathrm{m}$, the diameter of white air hole of $d_{1}$ is $0.6 \mu \mathrm{m}$, and the diameter of large air hole with the metallic layer of $d_{2}$ is $0.8 \mu \mathrm{m}$. RI of liquid analyte is increased from 1.40 to 1.42 , and RI of air is set as 1. The FEM mesh is used to separate the physical field for calculation as shown in Fig. 1(b). The outermost boundary of this AF-PCF is assigned with scattering boundary condition. The differences between two metal layers and a metal layer contained within this design are examined with more details. It exposes that the loss with one metal layer at the resonance wavelength is a lot less than that with two metal layers, while the resonance wavelength with two metal layers in the designed AF-PCF is not shifted compared to the resonance wavelength with one metal layer. A perfectly matched layer (PML) with several micrometers is supplied to the outmost layer that is used in the radiation power shielding. The design presented here focused on two key advantages. First, increasing the sensitivity the high refractive index of the liquid, since the central liquid channel is introduced. Second, as the designed AFPCF is simple and symmetrical, we are able to draw effortlessly after drawing tower by an appropriate temperature. Next, we would be capable of coating the metal film in the designed AF-PCF by the gas-phase condensation technique because gold has a lower boiling point than the melting point of silicon glass [5], [6], [8], [11], [12], [13], [14], [16], [18], [22]. 


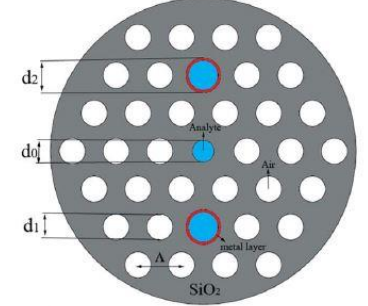

(a)

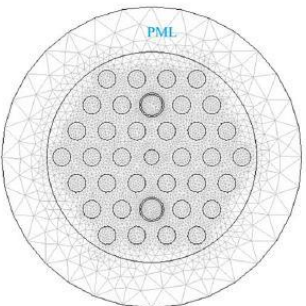

(b)
Fig. (6): (a) cross section of designed SPR sensor with gold and silver layers. (b) FEM mesh and scattering boundary condition for computation [55].

The coupled-mode process between the fundamental mode and the second SPP mode in this designed AF-PCF has been mathematically simulated with gold or silver layer, as seen in Fig. 7 [55].

\subsection{Design of a dual photonic crystal fiber with metal wire}

A new DC-PCF structure occupied with a metal wire (silver) in the center shown as red in Fig.8(a). There are many methods available to comprehend this structure, i.e., choosing a particular way to fill a metal wire into a conventional dielectric DC-PCF, such as pressureassisted pumping, or using femtosecond laser micromachining [77]. Fig. 8(b) is the simulation model, $\mathrm{d}_{\text {hole }}=2 \mu \mathrm{m}, \wedge$ $=3.75 \mu \mathrm{m}$. Depending on the available DC-PCF, structural parameters were chosen. The two cores are known as $\mathrm{A}$ and $\mathrm{B}$ for easy illustration. The dielectric characteristic of silica is described by the
Sellmeier equation [78] and silver by the Drude model [79]. The simulation has been solved using the FemSIM software to find the complex propagation constants of the waveguide modes with the finiteelement method. A perfectly matched layer (PML) also been supplied to properly evaluation the fiber confinement loss [80].
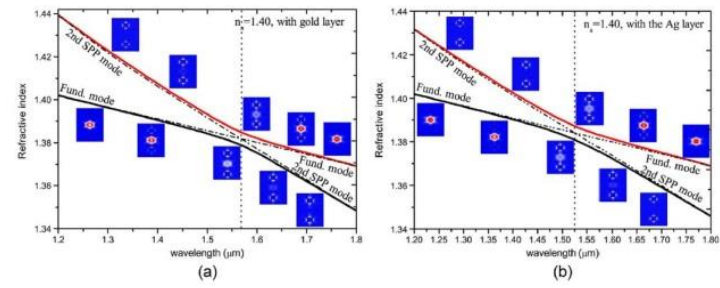

Fig. (7): (a) Wavelength dependence on the dispersion relations of the fundamental mode and the second-order SPP mode in this designed AF-PCF with the gold layer. (b) Wavelength dependence on the dispersion relations of the fundamental mode and the second-order SPP mode in this designed AFPCF with the silver layer. The analyte RI of $n_{a}$ is equal to 1.40. (Inserts) Electric field distributions of two coupled modes at a specific wavelength [55].
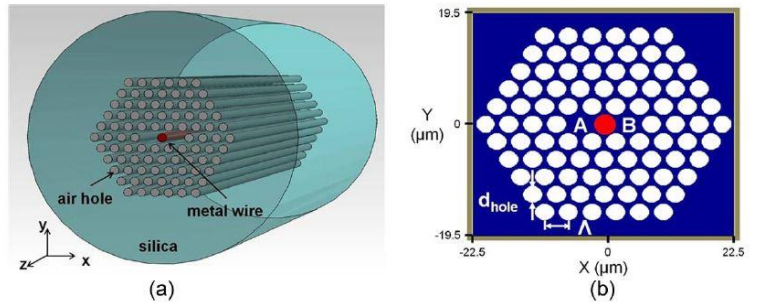

Fig. (8): (a) A new DC-PCF structure filled with a metal wire in the center (red). (b) The simulation model of the new structure [80].

\subsection{Design of plasmonic-photonic}

\section{crystal coupled nanolaser}


The basics of hybrid cavity structure are illustrated in Fig. 9. It involves two elements: a high-Q PC microcavity, that has been defined and studied in previous works [81] and a bowtie nano-antenna. The PC microcavity is designed to give a high-Q mode (5800) at telecom wavelength (Fig. 9(c)). Using 3D finitedifference time domain method (3DFDTD) with perfectly matched layers boundary conditions, the optical response of the structure was modelled. The nanocavity is excited with an oscillating dipole (pulsed temporal regime) appropriately oriented and positioned in the structure. The computational meshes for $\mathrm{x}, \mathrm{y}$, and $\mathrm{z}$ directions were $10 \mathrm{~nm}$. The evaluated intensity distributions of the $E_{x}$ and components of the field as shown in Fig. 9(b). Particularly, they revealed that the maximum of $E_{x}$ and $E_{y}$ do not overlap spatially: $E_{x}$ maximum are presented besides the cavity and $\mathrm{E}_{\mathrm{y}}$ maximum in the center of the cavity. This argument is crucial for the design of the hybrid structure [82], as will be discussed ahead. The optical bowtie antenna (NA) contains two coupled gold triangles separated by a nanometer size gap (20 nm) (Fig. 9(d)). To provide good wavelength matching between the resonant mode of the optical
NA and the PC the geometrical parameters had to be enhanced. As indicated in the FDTD simulations shown in Fig. 9(e), when the polarization of NA aligned along the gap it will strongly restricts the electromagnetic field in the gap, this direction will be stated as NA axis (Oy in Fig. 9) [87].

The additional field component polarized vertically to the NA axis is distributed at the four external corners of the bowtie triangles. On the contrary to the PC cavity mode spectrum, the NA resonant mode spectrum is broad, which allows an easy spectral overlap between the modes of each resonator. Two modes must coincide spectrally as well as spatially in order to improve the optical coupling between the fundamental mode of the microcavity and the NA resonance mode, in position and polarization. Theoretical calculations managed to determine the appropriate possible locations for the NA. Three of them have been chosen and are presented in Fig. 9. Several positions are possible, as shown in Fig. 9: two optimal cases of hybrid structures are revealed in Fig. 9(a) and 9(b), where the polarization of the field is aligned with the y direction (the NA is in the center of the cavity), or aligned with 
the $\mathrm{x}$ direction (the NA at the edge of the cavity), respectively. In both cases, the NA is located precisely at a field maximum of the photonic mode. An orthogonal example for the polarizations of the NA and of the optical mode is shown in Fig. 9(c).

In Fig. 9(a) and 9(b), the field of the resultant mode is highly restricted inside the NA gap, which reveals that there is an efficient coupling between the NA and the photonic cavity and strong spatial redistribution of the mode. On the contrary, in Fig. 9(c), one can see that the field intensity at the edges of the NA and spatial confinement is less efficient. This conclusion demonstrates that precise positioning and alignment of the NA inside the photonic cavity is absolutely necessary to obtain the anticipated coupling conditions between the two structures [83].

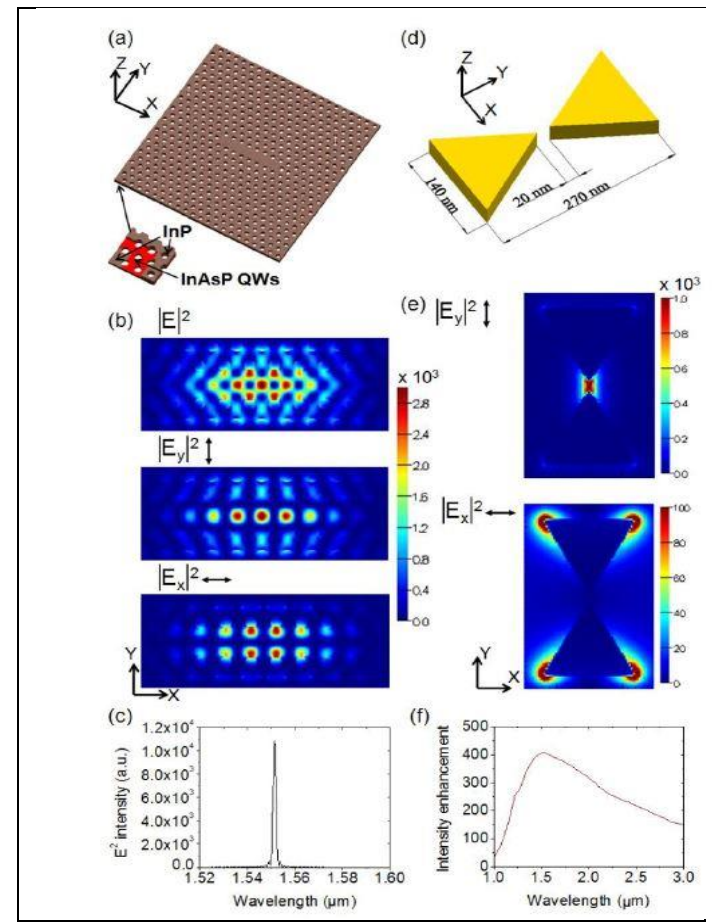

Fig. (9): Basic building blocks illustrations of the hybrid cavity. (a) Active photonic crystalbased linear microcavity : 4 InAsP QWs are located in the InP membrane (b) Calculated electric field intensity distribution $|\mathbf{E}|^{2},\left|\mathbf{E}_{\mathrm{x}}\right|^{2}$, and $\left|E_{y}\right|^{2}$ inside the nanocavity at the resonant wavelength of the fundamental mode $(\lambda=1.55$ $\mu \mathrm{m})$ (c) Spectrum of the nanocavity mode (d)

Artistic view of a gold Bow-tie nanoantenna(NA) (e) Calculated electric field intensity distribution $\left|E_{y}\right|^{2}$ and $\left|E_{x}\right|^{2}$ of the resonant mode of the NA, (f) Spectrum of the NA [84].

\section{Plasmonic PCF designs for various applications}

To attain desirable properties, e.g. sensitivity, detection range, stability, miniaturization etc., different models of plasmonic PCF structures were developed. For a diverse variety of applications namely refractive index (RI) measurement, double or multi-analyte 
sensing, temperature sensing, dual parameter sensing, biosensing, polarizing devices and couplers [84].

\subsection{D-Shaped Photonic Crystal Fiber with Rectangular Lattice and Nanoscale Gold Film.}

A D-shaped photonic fiber refractive index sensor with rectangular lattice focused on surface plasmon resonance was introduced and examined by Guowen An et al. The nanoscale gold-metal film is deposited in this kind of sensor on the flat surface in which it is side coated. Using the COMSOL Multiphysics program, a high-sensitivity refractive index sensor built on a D-shaped photonic crystal fiber with rectangular lattice and nanoscale gold film was evaluated via FEM. Mathematical findings show that the average sensitivity in the dynamic index range from 1.35 to 1.41 can be attained at $8.129 \mathrm{~nm} / \mathrm{RIU}$, and in the range from 1.31 to 1.35 the sensitivity can also exceed $2.000 \mathrm{~nm} /$ RIU. In particular, the typical sensitivity in the range from 1.35 to 1.41 is extraordinarily high compared with most of the fiber sensors recorded. In the meantime, despite its easy production cycle, the fiber sensor they have proposed can be a viable candidate for fiber biosensing implementations [85], [94].

\subsection{Ultra-high tunable photonic crystal fiber (PCF)}

Mohamed Farhat O. Hameed et al, researched and analyzed an innovative polarization filter for ultra-high tunable photonic crystal fiber (PCF) using FEM. The concept proposed has a core hole infiltrated with a noematic liquid crystal (NLC) that provides high temperature and external electric field tunability. In addition, the PCF is specifically packed into cladding air holes with metal wires. The statistical results indicate that the suggested filter is highly temperature-and external electric field tweakable [86].

\subsection{Photonic Crystal Fiber Biosensor}

A. A. Rifat et al. introduced a basic, two-ring Photonic Crystal Fiber (PCF) hexagonal lattice biosensor leveraging Surface Plasmon Resonance (SPR) phenomenon. An effective plasmonic gold layer and the analyte (sample) are positioned outside of the fiber structure rather than within the air-holes, allowing in a simpler and transparent manufacturing process. The sensor proposed demonstrates birefringent 
behaviour, which increases its sensitivity.

Finite Element Method (FEM) performs numerical examination of the guiding properties and the sensing efficiency. The sensor architecture suggested shows impressive results that could be used in the identification of biological and biochemical analytes $[4,8,16,22,36,87$, 97, 98].

\subsection{Coupling Plasmon Modes on Submicron Gold Wire in (PCF)}

H. W. Lee et al. report experimental results on the coupling to surface plasmon modes with gold nanowires specifically inserted into polarizationdependent photonic crystal wavelength transmission. In one sample the fibers are $24.5 \mathrm{~mm}$ long. Strongly polarized-and on-and off-resonance propagation ranges by as much as $45 \mathrm{~dB}$. Near-field optical images of light emanating from such a gold-filled fiber show light at surface plasmon resonances, directed on the fiber. Simulations of finite elements are in strong accordance with the test results. Such gold-filled fibers can presumably be used as filters and polarizers in fiberdependent wavelengths and as near-field tips for sub-wavelength imaging [7, 35, 96,98].

\subsection{Spiral photonic crystal fiber (PCF) with elliptic core}

A. M. Heikal et al, developed and analyzed a new, specifically metal filled photonic spiral crystal fiber (PCF) with elliptic center. The cladding air holes of the proposed model are located in spiral arms arranged elliptically. The dispersion properties and loss spectra of the stated prototype are studied using the full vectorial finite element method (FVFEM). In term of polarization dependent coupling between $\mathrm{x}$ and $\mathrm{y}$ - polarized core modes and surface plasmon polariton (SSP) are notably higher in the proposed design. Hence, making the structure appropriate for filter-based applications [88].

\subsection{Dual-core plasmonic photonic crystal fiber polarization $[97,98]$}

Zhenkai Fan et al, proposed two types of novel polarizing beam splitters dependent on dual-core photonic crystal fiber (DC-PCF) which have been developed and examined using the fullvector finite element method. The metallic gold is used as an active metal for the surface plasmon resonance (SPR). The resonant coupling between the second-order polariton surface plasmon 
(SPP) mode and the core-guided modes can improve the coupling properties of the built DCPCF. A gold layer is inserted in the designed DC-PCF to ensure optimal splitting efficiency and ultrabandwidth in contrast to the planned DCPCF with gold wire [89], [94].

\section{Conclusions}

In this review paper, nanophotonics technology has been presented as a confinement of electromagnetic fields on a subwavelength scale and surface plasmons plays a major role in advancing this arising field. It has been shown that, they may take various forms, ranging from freely propagating electron density waves along metal surfaces to localized electron oscillations on metal nanoparticles. The appealing characteristics could be illustrated by interaction between the free electrons' oscillations and electromagnetic waves of light gives the surface plasmons. The ability of light confinement and propagation through subwavelength structures grants the construction of photonic devices with minimum size, hence the characterize and design of plasmonic devices can be resolved using numerical simulation. In this review starts with general introduction about
Plasmonic photonic crystal fiber. This is followed by a historical background and literature review of Plasmonic PCF. Some types of designs are illustrated. Last of all, the applications of Plasmonic PCF are mentioned dependent on the types of design including the circular plasmonic photonic crystal fiber technique.

\section{References}

1- Qiang Liu b, Shuguang Li a,*, Xinyu Gao a, Highly sensitive plasmonics temperature sensor based on photonic crystal fiber with a liquid core, Key Laboratory of Metastable Materials Science and Technology, Key Laboratory for Microstructural Material Physics of Hebei Province, School of Science, Yanshan University, Qinhuangdao, 066004, PR China b School of Control Engineering, North Eastern University at Qinhuangdao, 066000, Optics Communications 427 (2018) 622-627, China

2- Mohammad Azab, M.F.O. Hameed Abed Nasr S. S. Obayya, Multifunctional Plasmonic Photonic Crystal Fiber Biosensors, January 2019 DOI: 10.1007/978-3-319-765563_10, In book: Computational Photonic Sensors,

3- Chao Liua, Guanglai Fua, Ex-centric core photonic crystal fiber sensor with gold nanowires based on surface plasmon resonance, a School of Electronics Science, Northeast Petroleum University, Daqing 163318, 
PR, Optik - International Journal for Light and Electron Optics 196 (2019) 163173, China.

4- Junbo Loua,b, Tonglei Chenga, Shuguang Lia, Xuenan Zhangaa, Surface plasmon resonance photonic crystal fiber biosensor based on goldgraphene Layers, State Key Laboratory of Synthetical Automation for Process Industries, College of Information Science and Engineering, Northeastern University, Shenyang 110819, Chinab Institute of Engineering and Technology, Tie Ling Normal College, Tieling 112000, Optical Fiber Technology 50 (2019) 206211, China.

5- Authors: Alok Kumar Paul, Ajay Krishno Sarkar, Md. Hafizul Islam, Monir Morshed Paul AK, Sarkar AK, Islam MH, Morshed M, Dual Core Photonic Crystal Fiber based Surface Plasmon Resonance Biosensor, Dual Core Photonic Crystal Fiber based Surface Plasmon Resonance Biosensor, Optik (2018), https://doi.org/10.1016/j.ijleo.2018.05 .131

6- K. Prabua, R. Malavikab, Highly birefringent photonic crystal fiber with hybrid cladding, Department of Electronics and Communication Engineering, National Institute of Technology Karnataka, Surathkal, India b School of Electronics Engineering, Vellore Institute of Technology, Vellore, Optical Fiber Technology 47 (2019) 21-26, India

7- A. A. Rifat*, G. Amouzad Mahdiraji, Y. G. Shee, Md. Jubayer Shawon, F.
R. Mahamd Adikan, A Novel Photonic Crystal Fiber Biosensor Using Surface Plasmon Resonance, Integrated Lightwave Research Group, Department of Electrical Engineering, Faculty of Engineering, University of Malaya, Kuala Lumpur-50603, Procedia Engineering 140 ( 2016 ) 1 7, Malaysia.

8- A. Rifat, Rajib Ahmed, G. Amouzad Mahdiraji and F. R. Mahamd Adikan, Highly Sensitive D-Shaped Photonic Crystal Fiber Based Plasmonic Biosensor in Visible to Near-IR Ahmmed, This article has been accepted for publication in a future issue of this journal, but has not been fully edited. Content may change prior to final publication. Citation information: DOI 10.1109/JSEN.2017.2677473, IEEE Sensors Journal

9- Abu Bakar Siddika, Selim Hossaina, Alok Kumar Paula, Mahabubur Rahmanb, Aslam Mollahc, High sensitivity property of dual-core photonic crystal fiber temperature sensor based on surface plasmon resonance, Sensing and Bio-Sensing Research $29 \quad$ (2020) 100350. Department of Electronics \& Telecommunication Engineering, Rajshahi University of Engineering \& Technology, Rajshahi 6204, Bangladesh.

10- Lee, H. W., Schmidt, M. A., Tyagi, H. K., Sempere, L. P., \& Russell, P. S. J. (2008). Polarization-dependent coupling to plasmon modes on submicron gold wire in photonic 
crystal fiber. Applied Physics

Letters, 93(11), 111102.

11-Zhao Yang b, Li Xia a,b, ${ }^{*}$, Chen Li b, Xin Chen b, Deming Liu a,b, A surface plasmon resonance sensor based on concave-shaped photonic crystal fiber for low refractive index detection, Optics Communications 430 (2019) 195-203, College of Optoelectronic Science and Engineering, Huazhong University of Science \& Technology, No.1037 Luoyu Road, Wuhan, Hubei 430074, China

12-Zhang, Jianshe Li*, Shuguang Li, Qiang LiuState, Broadband core shift photonic crystal fiber polarization filterat $1.55 \_\mathrm{m}$ based on surface plasmon resonanceShuhuan, Key Laboratory of Metastable Materials Science \& Technology and Key Laboratory for Microstructural Material Physics of HebeiProvince, School of Science, Yanshan University, Qinhuangdao, 066004, PR, Optik 165 (2018) 218-225, China

13-Araf Shafkat, Analysis of a gold coated plasmonic sensor based on a duplex core photonic crystal fiber, Department of Electrical \& Electronic Engineering (EEE), Chittagong University of Engineering \& Technology (CUET), Chittagong, Sensing and Bio-Sensing Research 28 (2020) 100324, Bangladesh

14- Authors:E. Rodr'iguez-Schwendtner, N. D'1az-Herrera, M.C. Navarrete, A. Gonza'lez-Cano, O' . Esteban E.Rodr'1guez-Schwendtner, N.D'iazHerrera,M.C.Navarrete,A.Gonza'lez-
Cano, $\mathrm{O}^{\prime}$.Esteban, Plasmonic sensor based on tapered optical fibers and magnetic fluids for measuring magnetic fields, Plasmonic sensor based on tapered optical fibers and magnetic fluids for measuring magnetic fields, Sensors andActuators:APhysicalhttp://dx.doi. org/10.1016/j.sna.2017.07.040

15- Ren, G., Shum, P., Yu, X., Hu, J., Wang, G., \& Gong, Y. (2008). Polarization dependent guiding in liquid crystal filled photonic crystal fibers. Optics communications, 281(6), 1598-1606.

16- Sujan Chakma, Md Abdul Khalek, Bikash Kumar Paul, Kawsar, Ahmed, Md Rabiul Hasan, Ali Newaz Bahar, Sujan Chakma, Md Abdul Khalek, Bikash Kumar Paul, Kawsar Ahmed, Md Rabiul Hasan, Ali Newaz Bahar, Gold-coated photonic crystal fiber biosensor based on surface, plasmon resonance: Design and analysis, Gold-coated photonic crystal fiber biosensor based on surface plasmon resonance: Design and analysis. The address for the corresponding author was captured as affiliation for all authors. Please check if appropriate. Sbsr(2018),doi:10.1016/j.sbsr.2018.0 2.003

17- Li, P., Zhao, J., \& Zhang, X. (2010). Nonlinear coupling in triangular triple-core photonic crystal fibers. Optics express, 18(26), 2682826833.

18-Esam A.A. Hagrasa,b, Mohamed Farhat O. Hameedb,c,d, A.M. 
Heikalb,e, S.S.A. Obayyab,e, Multifunctional photonic crystal fiber splitter for the two communication Bands, Optical Fiber Technology 52 (2019) 101986, journal homepage: www.elsevier.com/locate/yofte

19- Fang, X., Hu, M., Xie, C., Song, Y., Chai, L., \& Wang, C. (2011). High pulse energy mode-locked multicore photonic crystal fiber laser. Optics letters, 36(6), 1005-1007.

20- Hou, J., Bird, D., George, A., Maier, S., Kuhlmey, B. T., \& Knight, J. C. (2008). Metallic mode confinement in microstructured fibres. Optics Express, 16(9), 59835990.

21- Schmidt, M. A., Sempere, L. P., Tyagi, H. K., Poulton, C. G., \& Russell, P. S. J. (2008). Waveguiding and plasmon resonances in twodimensional photonic lattices of gold and silver nanowires. Physical Review B, 77(3), 033417.

22- Firoz Haider, Rifat Ahmmed Aoni, Rajib Ahmed, Md. Saiful Islam, and Andrey E. Miroshnichenko Propagation Controlled Photonic Crystal Fiber Based Plasmonic Sensor via Scaled-Down Approach, This article has been accepted for publication in a future issue of this journal, but has not been fully edited. Content may change prior to final

23- publication. Citation information: DOI 10.1109/JSEN.2018.2880161, IEEE Sensors Journal

24- Lee, H. W., Schmidt, M. A., Russell, R. F., Joly, N. Y., Tyagi, H. K., Uebel, P., \& Russell, P. S. J.
(2011). Pressure-assisted melt-filling and optical characterization of $\mathrm{Au}$ nano-wires in microstructured fibers. Optics Express, 19(13), 1218012189.

25- Md. Mahabubur Rahman1, Md. Masud Rana2, M. S. Anower2, M. Saifur Rahman2, Alok Kumar Paul2, Received: 3 March 2020 / Accepted: 30 May 2020/ Design and analysis of photonic crystal fber-based plasmonic, microbiosensor: an external sensing scheme, / Published online: 10 June 2020, Springer Nature Switzerland AG 2020

26- Heikal, A. M., Hussain, F. F. K., Hameed, M. F. O., \& Obayya, S. S. (2015). Efficient polarization filter design based on plasmonic photonic crystal fiber. Journal of Lightwave Technology, 33(13), 2868-2875.

27- Nagasaki, A., Saitoh, K., \& Koshiba, M. (2011). Polarization characteristics of photonic crystal fibers selectively filled with metal wires into cladding air holes. Optics express, 19(4), 3799-3808.

28- Lee, H. W., Schmidt, M. A., \& Russell, P. S. J. (2012). Excitation of a nanowire "molecule" in gold-filled photonic crystal fiber. Optics letters, 37(14), 2946-2948.

29- Li, P., \& Zhao, J. (2013). Polarization-dependent coupling in gold-filled dual-core photonic crystal fibers. Optics Express, 21(5), 52325238 .

30- Jian Hana, Weiquan Sua, Chao Liua,, Famei Wangb, Chunhong Xua, Qiang Liua, Lin Yanga, Xianli Lia, Tao Sunc, Paul K. Chud,Asymmetrical 
photonic crystal fiber based on the surface plasmon, resonance sensor and analysis by the lowerbirefringence peak, method, Optik International Journal for Light and Electron Optics 189 (2019) 121-129

31-Md. Rabiul Hasan, Sanjida Akter, Kawsar Ahmed, and Derek Abbott, Plasmonic Refractive Index Sensor Employing Niobium Nanofilm on Photonic Crystal Fiber, Fellow, IEEE This article has been accepted for publication in a future issue of this journal, but has not been fully edited. Content may change prior to final publication. Citation information: DOI 10.1109/LPT.2017.2786475, IEEE Photonics Technology Letters

32- Krasavin, A. V., \& Zayats, A. V. (2010). Electro-optic switching element for dielectric-loaded surface plasmon polariton waveguides. Applied Physics Letters, 97(4), 041107.

33- Bilotti, F., Tricarico, S., \& Vegni, L. (2009). Plasmonic metamaterial cloaking at optical frequencies. IEEE Transactions on Nanotechnology, 9(1), 55-61.

34- Chen, C. H., \& Liao, K. S. (2013). $1 \mathrm{xN}$ plasmonic power splitters based on

metal-insulator-metal waveguides. Optics express, 21(4), 4036-4043.

35-Md. Rabiul Hasan, Student Member, IEEE, Sanjida Akter, Ahmmed A. Rifat, Sohel Rana, Kawsar Ahmed, Rajib Ahmed, Harish Subbaraman, Member, IEEE, and Derek Abbott, Fellow, IEEE,Spiral Photonic Crystal
Fiber-Based Dual-Polarized Surface Plasmon Resonance Biosensor, IEEE SENSORS JOURNAL, VOL. 18, NO. 1, JANUARY 1, 2018

36- A. M. Heikal, Mohamed Farhat O. Hameed, Member, IEEE, Firas Faeq K. Hussain, S.S.A. Obayya, Senior Member, IEEE, Efficient Polarization Filter Design Based on Plasmonic Photonic Crystal Fiber, This article has been accepted for publication in a future issue of this journal, but has not been fully edited. Content may change prior to final publication. Citation information: DOI 10.1109/JLT.2015.2419175, Journal of Lightwave Technology

37- Das, S., Haldar, R., \& Varshney, S. K. (2013). Triple-core collinear and noncollinear plasmonic photonic crystal fiber couplers. Applied optics, 52(34), 8199-8204.

38- Varshney, S. K., Saitoh, K., Sinha, R. K., \& Koshiba, M. (2009). Coupling Characteristics of Multicore Photonic Crystal Fiber-Based 1\$l,times $\backslash, \quad \$ 4 \quad$ Power Splitters. Journal of Lightwave Technology, 27(12), 2062-2068.

39- Khan, K. R., \& Wu, T. X. (2008). Short pulse propagation in wavelength selective index-guided photonic crystal fiber coupler. IEEE Journal of Selected Topics in Quantum Electronics, 14(3), 752-757.

40- Deepmala Kulshreshth $\Uparrow$, Yazusha Sharma, Ashish Kulshrestha, Shruti Kalra, Laxmi Narayan Balai,Dispersion properties of silica based carbon disulphide filled 
photonic crystal fiber, Department of Electronics and Communication Engineering, YIT Jaipur, JECRC Jaipur, JECRC Jaipur , JECRC, Jaipur, YIT, Jaipur, India, Materials Today: Proceedings

41- Khan, K. R., Wu, T. X., Christodoulides, D. N., \& Stegeman, G. I. (2008). Soliton switching and multi-frequency generation in a nonlinear photonic crystal fiber coupler. Optics Express, 16(13), 9417-9428.

42- Uthayakumar, T., Raja, R. V. J., \& Porsezian, K. (2012). All-optical steering of light through nonlinear twin-core photonic crystal fiber coupler at $850 \mathrm{~nm}$. Journal of lightwave technology, 30(13), 21102116.

43- Yundong Liu, Xili Jing*, Shuguang Li, Shuhuan Zhang, Zhen Zhang, Ying Guo, Jie Wang, Shun Wang, High sensitivity surface plasmon resonance sensor based on D-shaped photonic crystal fiber with circular layout, State Key Laboratory of Metastable Materials Science \& Technology and Key Laboratory for Microstructural Material Physics of Hebei Province, School of Science, Yanshan University, Qinhuangdao 066004, PR, Optical Fiber Technology 46 (2018) 311-317, China

44- Saitoh, K., Sato, Y., \& Koshiba, M. (2003). Coupling characteristics of dual-core photonic crystal fiber couplers. Optics express, 11(24), 3188-3195.
45- Saitoh K, Sato Y, Koshiba M (2004) Polarization splitter in three core photonic crystal fibers. Opt Express 12:3940-3946

46- Chen D, Hu G, Chen L (2011) Dual-core photonic crystal fiber for hydrostatic pressure sensing. IEEE Photon Technol Lett 23:1851-1853.

47- A. A. Rifat, G. Amouzad Mahdiraji, Y. G. Shee, Md. Jubayer Shawon, F. R. Mahamd Adikan, A Novel Photonic Crystal Fiber Biosensor Using Surface Plasmon ResonanceIntegrated Lightwave Research Group, Department of Electrical Engineering, Faculty of Engineering, University of Malaya, Kuala Lumpur-50603, Malaysia, 2019.

48- Younis, B. M., Heikal, A. M., Hameed, M. F. O., \& Obayya, S. S. A. (2017). Coupling enhancement of plasmonic liquid photonic crystal fiber. Plasmonics, 12(5), 1529-1535.

49- Veselago, V. G. (1967). Electrodynamics of substances with simultaneously negative and. Usp. Fiz. Nauk, 92, 517.

50- Pendry, J. B. (2000). Negative refraction makes a perfect lens. Physical review letters, 85(18), 3966.

51- Johnson, P. B., \& Christy, R. W. (1972). Optical constants of the noble metals. Physical review B, 6(12), 4370.

52- Marton, J. P., \& Jordan, B. D. (1977). Optical properties of aggregated metal systems: Interband transitions. Physical Review B, 15(4), 1719.

53- West, P. R., Ishii, S., Naik, G. V., Emani, N. K., Shalaev, V. M., \& Boltasseva, A. (2010). Searching for 
better plasmonic materials. Laser \& Photonics Reviews, 4(6), 795-808.

54- Tan ZX, Hao X, Shao YH, Chen YZ, Li XJ, Fan P (2014) Phase modulation and structural effects in a D-shaped all-solid photonic crystal fiber surface plasmon resonance sensor. Opt Express 12:15049-15063.

55- Dash JN, Jha R (2014) Graphenebased birefringent photonic crystal fiber sensor using surface plasmon resonance. IEEE Photon Technol Lett 26:1092-1095.

56- Shan X, Patel U, Wang S, Iglesias $\mathrm{R}$, Tao N (2010) Imaging local electrochemical current via surface plasmon resonance. Science 3271:363-1366.

57- Steiner, G. (2004). Surface plasmon resonance imaging. Analytical and bioanalytical chemistry, 379(3), 328-331.

58- Geng P, Zhang W, Gao S, Zhang S, Zhang H, Ruan J (2012) Orthogonal single-polarization single-core photonic crystal fiber for wavelength splitting. IEEE Photon Technol Lett 24:1304- 1306.

59- Rosa L, Poli F, Foroni M, Cucinotta A, Selleri S (2006) Polarization splitter based on a square-lattice photonic-crystal fiber. Opt Lett 31:441-443.

60- Spittel, R., Schuster, D. H. K., Kobelke, J., \& Bartelt, H. (2011). Plasmonic effects in metal filled microstructured optical fibers. In $\mathrm{DGaO}$ Proceedings.

61- Fan, Z., Li, S., Liu, Q., An, G., Chen, H., Li, J., ... \& Tian, W. (2015). High sensitivity of refractive index sensor based on analyte-filled photonic crystal fiber with surface plasmon resonance. IEEE Photonics Journal, 7(3), 1-9.

62- Kao, K. C., \& Hockham, G. A. (1966, July). Dielectric-fibre surface waveguides for optical frequencies. In Proceedings of the Institution of Electrical Engineers (Vol. 113, No. 7, pp. 1151-1158). IET Digital Library.

63- Markos, C., Travers, J. C., Abdolvand, A., Eggleton, B. J., \& Bang, O. (2017). Hybrid photoniccrystal fiber. Reviews of Modern Physics, 89(4), 045003.

64- Knight, J. C., Birks, T. A., Russell, P. S. J., \& Atkin, D. M. (1996). Allsilica single-mode optical fiber with photonic crystal cladding. Optics letters, 21(19), 1547-1549.

65- Birks, T. A., Knight, J. C., \& Russell, P. S. J. (1997). Endlessly single-mode photonic crystal fiber. Optics letters, 22(13), 961-963.

66- Knight, J. C., Arriaga, J., Birks, T. A., Ortigosa-Blanch, A., Wadsworth, W. J., \& Russell, P. S. J. (2000). Anomalous dispersion in photonic crystal fiber. IEEE photonics technology letters, 12(7), 807-809.

67- Suzuki, K., Kubota, H., Kawanishi, S., Tanaka, M., \& Fujita, M. (2001). Optical properties of a low-loss polarization-maintaining photonic crystal fiber. Optics Express, 9(13), 676-680.

68- Yokouchi, N., Danner, A. J., \& Choquette, K. D. (2003). Twodimensional photonic crystal confined vertical-cavity surface-emitting lasers. IEEE Journal of Selected Topics in Quantum Electronics, 9(5), 1439-1445.

69- Borel, P. I., Frandsen, L. H., Thorhauge, M., Harpøth, A., Zhuang, 
Y. X., Kristensen, M., \& Chong, H. M. H. (2003). Efficient propagation of TM polarized light in photonic crystal components exhibiting band gaps for TE polarized light. Optics express, 11(15), 1757-1762.

70- Yasuda, T., Tsuji, Y., \& Koshiba, M. (2004). Tunable light propagation in photonic crystal coupler filled with liquid crystal. IEEE photonics technology letters, 17(1), 55-57.

71- Hassani, A., \& Skorobogatiy, M. (2006). Design of the microstructured optical fiber-based surface plasmon resonance sensors with enhanced microfluidics. Optics Express, 14(24), 11616-11621.

72- Di Teodoro, F., \& Brooks, C. D. (2007, February). Multi-MW peak power, single transverse mode operation of a 100 micron core diameter, Yb-doped photonic crystal rod amplifier. In Fiber Lasers IV: Technology, Systems, and Applications (Vol. 6453, p. 645318). International Society for Optics and Photonics.

73- Chien, H. T., Lee, C., Chiu, H. K., Hsu, K. C., Chen, C. C., Ho, J. A. A., \& Chou, C. (2009). The comparison between the graded photonic crystal coupler and various couplers. Journal of Lightwave Technology, 27(14), 2570-2574.

74- Chen, L., Zhang, W., Zhang, Z., Liu, Y., Sieg, J., Zhang, L., ... \& Yan, T. (2014). Design for a singlepolarization photonic crystal fiber wavelength splitter based on hybridsurface plasmon resonance. IEEE photonics journal, 6(4), 1-9.

75- Khaleque, A., Mironov, E. G., \& Hattori, H. T. (2015). Analysis of the properties of a dual-core plasmonic photonic crystal fiber polarization splitter. Applied Physics B, 121(4), 523-532.

76- Ng, W. L., Rifat, A. A., Wong, W. R., Tee, D. C., \& Mahamd Adikan, F. R. (2017). Enhancement of evanescent field exposure in a photonic crystal fibre with interstitial holes. Journal of Modern Optics, 64(15), 1544-1549.

77- Chakma, S., Khalek, M. A., Paul, B. K., Ahmed, K., Hasan, M. R., \& Bahar, A. N. (2018). Gold-coated photonic crystal fiber biosensor based on surface plasmon resonance: design and analysis. Sensing and bio-sensing Research, 18, 7-12.

78- Azman, M. F., Mahdiraji, G. A., Wong, W. R., Aoni, R. A., \& Adikan, F. R. M. (2019). Design and fabrication of copper-filled photonic crystal fiber based polarization filters. Applied optics, 58(8), 20682075.

79- Agrawal, G. P. (2007). Nonlinear Fiber Optics (Academic Press, New York, USA).

80- Vial, A., Grimault, A. S., Macías, D., Barchiesi, D., \& de La Chapelle, M. L. (2005). Improved analytical fit of gold dispersion: Application to the modeling of extinction spectra with a finite-difference time-domain method. Physical Review B, 71(8), 085416.

81- Florous, N., Saitoh, K., \& Koshiba, M. (2005). A novel approach for designing photonic crystal fiber splitters with polarization-independent propagation characteristics. Optics express, 13(19), 7365-7373.

82- Jiang, H., Wang, E., Zhang, J., Hu, L., Mao, Q., Li, Q., \& Xie, K. (2014). 
Polarization splitter based on dualcore photonic crystal fiber. Optics express, 22(25), 30461-30466.

83- Wang, Y., Liao, C. R., \& Wang, D. N. (2010). Femtosecond laser-assisted selective infiltration of microstructured optical fibers. Optics express, 18(17), 18056-18060.

84- Verma, R. K., \& Gupta, B. D. (2008). Theoretical modelling of a bidimensional U-shaped surface plasmon resonance based fibre optic sensor for sensitivity enhancement. Journal of Physics D: Applied Physics, 41(9), 095106.

85- Sharma, A. K., \& Gupta, B. D. (2007). Influence of dopants on the performance of a fiber optic surface plasmon resonance sensor. Optics communications, 274(2), 320-326.

86- Zhang, S., Yu, X., Zhang, Y., Shum, P., Zhang, Y., Xia, L., \& Liu, D. (2012). Theoretical study of dualcore photonic crystal fibers with metal wire. IEEE photonics Journal, 4(4), 1178-1187.

87- Le Gac, G., Rahmani, A., Seassal, C., Picard, E., Hadji, E., \& Callard, S. (2009). Tuning of an active photonic crystal cavity by an hybrid silica/silicon near-field probe. Optics express, 17(24), 21672-21679.

88- Akahane, Y., Asano, T., Song, B. S., \& Noda, S. (2003). High-Q photonic nanocavity in a twodimensional photonic crystal. Nature, 425(6961), 944-947.

89- Zhang, T., Callard, S., Jamois, C., Chevalier, C., Feng, D., \& Belarouci, A. (2014). Plasmonic-photonic crystal coupled nanolaser. Nanotechnology, 25(31), 315201.
90- Hu, D. J. J., \& Ho, H. P. (2017). Recent advances in plasmonic photonic crystal fibers: design, fabrication and applications. Advances in Optics and Photonics, 9(2), 257-314.

91- An, G., Li, S., Qin, W., Zhang, W., Fan, Z., \& Bao, Y. (2014). Highsensitivity refractive index sensor based on D-shaped photonic crystal fiber with rectangular lattice and nanoscale gold film. Plasmonics, 9(6), 1355-1360.

92- Hameed, M. F. O., Heikal, A. M., Younis, B. M., Abdelrazzak, M., \& Obayya, S. S. A. (2015). Ultra-high tunable liquid crystal-plasmonic photonic crystal fiber polarization filter. Optics express, 23(6), 70077020.

93- Rifat, A. A., Mahdiraji, G. A., Sua, Y. M., Shee, Y. G., Ahmed, R., Chow, D. M., \& Adikan, F. M. (2015). Surface plasmon resonance photonic crystal fiber biosensor: a practical sensing approach. IEEE Photonics Technology Letters, 27(15), 16281631.

94- Fan, Z., Li, S., Liu, Q., Li, J., \& Xie, Y. (2015). Plasmonic polarization beam splitter based on dual-core photonic crystal fiber. Plasmonics, 10(6), 1283-1289.

95- Junbo Loua,b, Tonglei Chenga, Shuguang Lia, Xuenan Zhanga, Surface plasmon resonance photonic crystal fiber biosensor based on goldgraphene Layers, Optical Fiber Technology 50 (2019) 206-211

96- Moriom Rojy Momota, Md. Rabiul Hasan, Rajshahi, Hollow-core silver 
MJPS, VOL.(7), NO.(2), 2020

coated photonic crystal fiber plasmonic sensor, University of Engineering \& Technology, Department of Electronics \& Telecommunication Engineering, Rajshahi, 6204, Bangladesh, Optical Materials 76 (2018) 287e294

97-Ahmmed A. Rifat, G. Amouzad Mahdiraji, Rajib Ahmed, Desmond M. Chow, Y. M. Sua, Y. G. Shee, F. R. Mahamd Adikan, CopperGraphene-Based Photonic Crystal Fiber Plasmonic Biosensor, Volume 8, Number 1, February 2016, DOI: 10.1109/JPHOT.2015.251063219430655 Ó 2015 IEEE

98- A. A. Rifat, G. Amouzad Mahdiraji, Y. G. Shee, Md. Jubayer Shawon, F. R. Mahamd Adikan, RS Singapore - ICMAT Symposia Proceedings, 8th International Conference on Materials for Advanced Technologies A Novel Photonic Crystal Fiber Biosensor Using Surface Plasmon Resonance, Integrated Lightwave Research Group, Department of Electrical Engineering, Faculty of Engineering, University of Malaya, Kuala Lumpur50603, Malaysia, 2020. 